\title{
Experimental Study of Methane Heat Transfer Deterioration in a Subscale Combustion Chamber
}

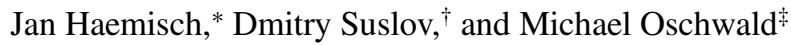 \\ German Aerospace Center (DLR), 74239 Hardthausen, Germany
}

DOI: $\underline{10.2514 / 1 . B 37394}$

\begin{abstract}
Methane has many possible advantages as a fuel for reusable rocket engines and is therefore extensively discussed. However, the characteristics of methane as a coolant in a regenerative cooling system are widely unknown. Especially the heat transfer deterioration that occurs in the vicinity of the critical point is a major concern and a severe drawback. To investigate this phenomenon and to establish a broad data basis for the validation of numerical simulations, tests were performed with a subscale combustion chamber. The combustion chamber contains different cooling channel geometries (rectangular cooling channels with the aspect ratios [height-to-width ratio]: 1.7, 3.5, 9.2, and 30) and was cooled with cryogenic methane. Results close to the critical point show a distinct maximum in hot gas wall temperature, which is a clear evidence of heat transfer deterioration. The test results indicate a strong dependency on the occurrence of this effect toward cooling channels with a low aspect ratio.
\end{abstract}

\section{Nomenclature}

$A R=$ channel aspect ratio

$b=$ cooling channel width, $\mathrm{mm}$

$C_{p} \quad=$ specific heat capacity at constant pressure, $\mathrm{J} / \mathrm{kg} / \mathrm{K}$

$D_{\mathrm{BK}} \quad=$ combustion chamber diameter, $\mathrm{mm}$

$d_{H} \quad=$ hydraulic diameter, $\mathrm{mm}$

$G \quad=$ specific mass flow rate, $\mathrm{kg} / \mathrm{s} / \mathrm{m}^{2}$

$h=$ cooling channel height, $\mathrm{mm}$

$h_{\text {in }} \quad=\quad$ enthalpy at the inlet, $\mathrm{kJ} / \mathrm{kg}$

$h_{\text {out }} \quad=$ enthalpy at the outlet, $\mathrm{kJ} / \mathrm{kg}$

$k_{s} \quad=\quad$ equivalent sand grain roughness, $\mathrm{m}$

$L_{\mathrm{HG}} \quad=\quad$ hot gas side width per cooling channel, $\mathrm{mm}$

$\dot{m} \quad=$ mass flow, $\mathrm{kg} / \mathrm{s}$

$n=$ number of cooling channels (per $360^{\circ}$ )

$P \quad=$ pressure, bar

$P_{\mathrm{cc}} \quad=$ combustion chamber pressure, bar

$P_{\mathrm{cr}} \quad=$ critical pressure, bar

$P_{\text {in }} \quad=$ inlet pressure, bar

$Q 1-Q 4=$ quadrant 1 to quadrant 4

$\dot{q}_{w} \quad=$ wall heat flux, $\mathrm{W} / \mathrm{m}^{2}$

$T_{i}^{\exp } \quad=$ measured temperature value, $\mathrm{K}$

$T_{i}^{\text {calc }}=$ calculated temperature value, $\mathrm{K}$

$T_{\mathrm{HG}}=$ hot gas side surface temperature, $\mathrm{K}$

$T_{\text {in }} \quad=\quad$ fluid inlet temperature, $\mathrm{K}$

$T_{\text {out }} \quad=\quad$ fluid outlet temperature, $\mathrm{K}$

$U=$ cooling channel circumference, $\mathrm{mm}$

$\dot{V}=$ volume flow, $\mathrm{m}^{3} / \mathrm{s}$

$\alpha \quad=$ heat transfer coefficient, $\mathrm{W} / \mathrm{m}^{2} / \mathrm{K}$

$\lambda=$ thermal conductivity, $\mathrm{W} / \mathrm{m} / \mathrm{K}$

\section{Introduction}

C HEMICAL rocket engines represent the only propulsion system that is able to transport payload into the Earth orbit. The combination of liquid oxygen (LOX) and liquid hydrogen (LH2) has been investigated since the 1960s and is used in many first- and upperstage engines (such as Vulcain, Vinci, SSME, and RD-0120). Until recently, only two engines have proved to be reusable: The Space Shuttle Main Engine (SSME) (LOX/LH2) and the Merlin engine (LOX/RP1). Nowadays methane is in discussion as a propellant for a new generation of reusable rocket engines because it may have advantages over both combinations [1,2].

In contrast to hydrogen, which enters the cooling channels at much higher temperatures and pressures than the critical values, methane enters the cooling channels at supercritical pressure but subcritical temperature. It is then heated up by the combustion chamber and crosses the Widom line, which is the extension of the coexisting line into the supercritical domain. The Widom line is defined as the connection of points where the specific heat at constant pressure has its maximum value [3]. In this area, sometimes also called pseudocritical temperature, the physical properties of a fluid are very much affected by small changes in pressure and temperature.

In this region the heat transfer can be normal, enhanced, or deteriorated (see Table 1 [4]). While the normal and enhanced heat transfer have only minor consequences and could only influence the engine performance, the heat transfer deterioration has a major impact due to a possible very high peak in hot gas side wall temperature [4-7].

With decreasing pressure, the specific heat capacity at constant pressure exhibits an increasing sharp maximum (see Figs. 1 and 2 ). In addition to a high heat flux, this can lead to a separation of hot-gaslike coolant at the wall and cold-liquid-like coolant in the bulk flow. This results in a locally decreasing heat transfer coefficient. This effect is known as heat transfer deterioration (HTD) and has been investigated in power plants since the 1960s [7]. It is nowadays intensively investigated numerically $[\underline{6}, \underline{8}]$ and experimentally $[\underline{9}, \underline{10}]$ mainly due to the impact on methane in rocket-engine-cooling channels [11]. Especially for reusable engines, where the pressure level is kept at lower values to protect the structure and in situations where the engine has to be throttled down, this effect could result in a catastrophic failure. However, the available experiments are limited to electrically heated tubes and until now no experimental data have been available for regenerative cooling channels at real conditions.

Following [ 8$]$ and [4] the occurrence of heat transfer deterioration is defined as the existence of a peak in hot gas side wall temperature at the same location where the heat transfer coefficient exhibits a minimum value. For a nondeteriorated flow for a constant heat flux, the hot gas side wall temperature is continuously rising. When it comes to deterioration, the gas-like layer at the wall limits the heat 
Table 1 The three possible types of heat transfer in the transcritical region [4]

\begin{tabular}{lcc}
\hline \hline Heat transfer & Hot gas side temperature & Heat transfer coefficient \\
\hline Normal & Monotonically rising & Monotonically rising \\
Enhanced & Monotonically rising & Local maximum \\
Deteriorated & Local maximum & Local minimum \\
\hline \hline
\end{tabular}

transfer and the hot gas side wall temperature is strongly rising. Because of the low density of this layer, the fluid velocity close to the wall increases. Further downstream this acceleration results in an M-shaped velocity profile that can be seen in numerical simulations $[\underline{6}, 13]$ and an increasing heat transfer coefficient at the cooling channel side. The hot gas side wall temperature decreases again. The occurrence of a peak in hot gas side wall temperature can experimentally be detected and is therefore a very good indicator whether heat transfer deterioration takes place or not.

\section{Discussion}

\section{A. Experimental Setup}

To study the heat transfer phenomena with methane as a coolant in regenerative cooling channels, tests were performed with a cylindrical research combustion chamber segment that is divided into four sections around the circumference, each containing certain cooling channel geometry (rectangular cooling channels with different aspect ratios [height-to-width-ratio]; see Fig. 3 and Table 2). The setup ensures the same mass flow for each sector and makes the different geometries comparable.

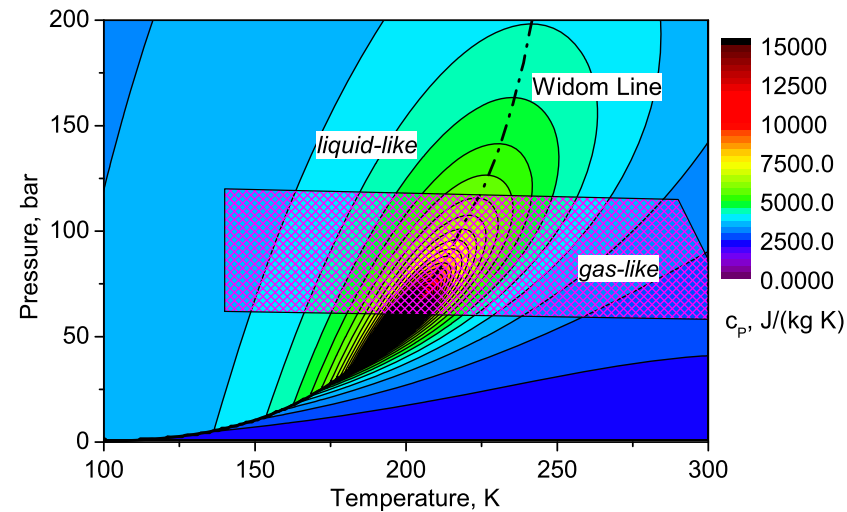

Fig. 1 Specific heat capacity at constant pressure for methane. Shaded area is covered by the tests, presented in this paper.

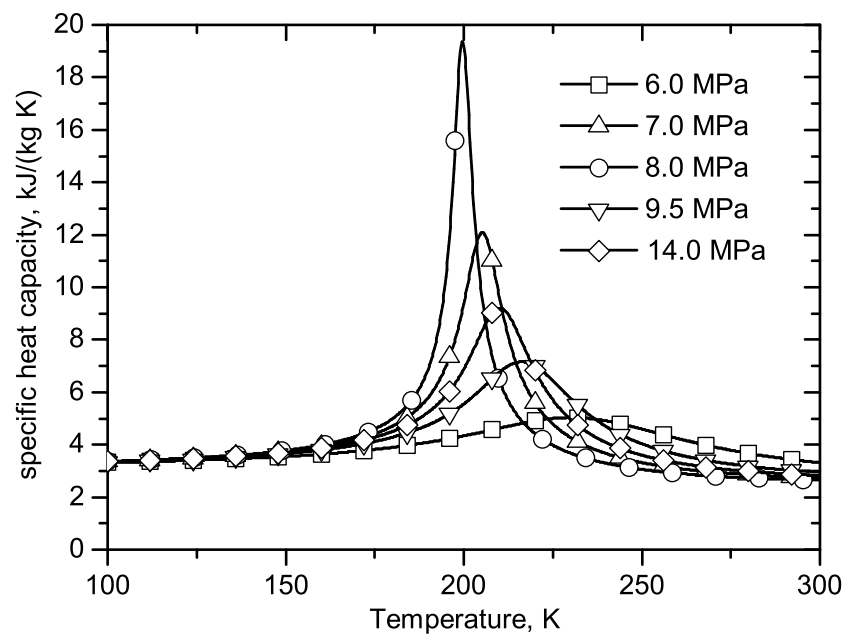

Fig. 2 Specific heat capacity at constant pressure and dynamic viscosity for methane as a function of temperature for different pressure values [12].
This segment, called the high-aspect-ratio cooling channels (HARCC) segment, is part of the combustion chamber " $D$ " that has an inner diameter of $D_{\mathrm{BK}}=80 \mathrm{~mm}$. The length of the combustion chamber segment used in this study is $0.2 \mathrm{~m}$, that is, 130-400 times the hydraulic diameter $d_{H}$ of the cooling channels (depending on the sector and thereby the aspect ratio). The segment is made of the copper alloy ELBRODUR ${ }^{\circledR}(\mathrm{CuCrZr})$. The cooling channels are wire cut into the liner and closed by electrodeposit copper and nickel. Before closing, the channels were highly polished. An equivalent sand grain roughness of $k_{s}=0.2 \mu \mathrm{m}$ was measured [14]. Thermal conductivity of the material was measured to be $\lambda=350 \mathrm{~W} /(\mathrm{m} \cdot \mathrm{K})$.

An even heat flux distribution on the HARCC segment is ensured with a large number of 42 coaxial injection elements and the standard segment that is placed between the HARCC segment and the injection head. The distribution of cooling fluids is sketched in Fig. 4 . The coolant mass flow for the HARCC segment is dumped and can therefore be controlled independently from the remaining combustion chamber. The mass flow for each sector is adjusted with orifices and measured separately by measurement turbines. The average maximum deviation between the sectors is $\pm 10 \%$.

At stable and constant conditions inside the combustion chamber, in other words, constant combustion chamber pressure and mixture ratio, the coolant mass flow of the HARCC segment is reduced stepwise down to a chosen limit without changing the hot gas conditions. Because of restrictions of the test bench that demand a single feed-line for the coolant and the propellant, the pressure inside the investigated cooling channels could not be controlled independently from the combustion chamber. Therefore a low coolant pressure goes along with a low pressure inside the combustion chamber.

Liquefied natural gas, which consists of $>98 \%$ methane, at a temperature as low as $130 \mathrm{~K}$, was used as cooling fluid. To investigate pseudoboiling effects, pressure inside the cooling channels was throttled up to a reduced pressure as low as $P_{\text {in }} / P_{\text {cr }}=1.2$. The thermal field was measured with 80 thermocouples, 20 per sector, integrated in the combustion chamber wall at 4 axial positions and 5 different distances to the combustion chamber wall (see Fig. $\underline{5}$ and Table 3). With an inverse temperature field method (Sec. II.B) it is possible to reconstruct the thermal field and to characterize the heat transfer behavior inside the entire cooling channel.

The tests were performed at the European Research and Technology Test Facility P8 in Lampoldshausen. This test facility enables investigations at typical rocket engine operating conditions. Conditions in these tests reached chamber pressures up to $65 \mathrm{bar}$, coolant pressure between 58 and 120 bar, and heat fluxes up to $\dot{q}_{w}=18 \mathrm{MW} / \mathrm{m}^{2}$.

Figure $\underline{6}$ shows a picture of the combustion chamber inside the test cell during a hot run.

\section{B. Inverse Method}

The aforementioned inverse method was implemented to obtain the local heat transfer properties $[\underline{15}, 16]$. The method is based on the local temperature measurements of the structure in between cooling

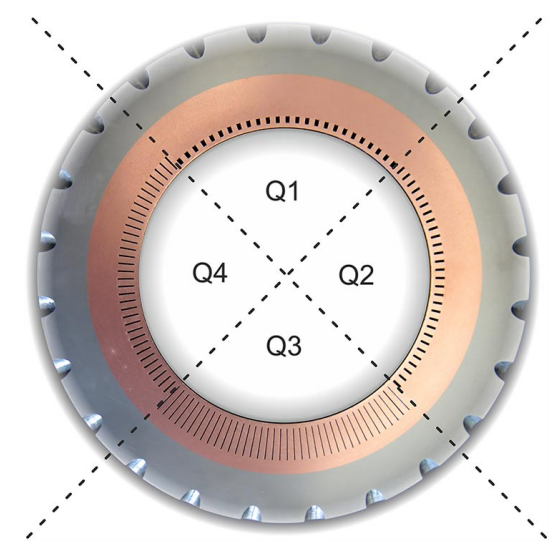

Fig. 3 Cut through the HARCC segment that reveals the 4 channel geometries. 
Table 2 Geometry of the investigated cooling channels

\begin{tabular}{|c|c|c|c|c|}
\hline Sector & $\mathrm{Q} 1$ & Q2 & Q3 & $\overline{\mathrm{Q} 4}$ \\
\hline Height, mm & 2 & 2.8 & 9 & 4.6 \\
\hline Width, mm & 1.2 & 0.8 & 0.3 & 0.5 \\
\hline Aspect ratio & 1.7 & 3.5 & 30 & 9.2 \\
\hline Distance to hot gas side, $\mathrm{mm}$ & 1.1 & 1.1 & 1.1 & 1.1 \\
\hline Distance between cooling channels, $\mathrm{mm}$ & 1.4 & 1.4 & 1.4 & 1.4 \\
\hline Area, $\mathrm{mm}^{2}$ & 2.4 & 2.24 & 2.7 & 2.3 \\
\hline Hydraulic diameter, $\mathrm{mm}$ & 1.5 & 1.24 & 0.58 & 0.9 \\
\hline No. of cooling channels (per $90^{\circ}$ ) & 25 & 29 & 38 & 34 \\
\hline
\end{tabular}

channels at steady-state thermal conditions; the temperatures are used to numerically reconstruct the thermal field. In the present study, the method is used to calculate the hot gas side wall temperature and the heat flux inside the cooling channels.

In the first step, the thermal field of the structure is calculated by a $3 \mathrm{D}$ finite element analysis with the assumed heat flux and heat transfer coefficient boundary conditions that are sketched in Fig. 7. Two parameters for heat flux and four parameters for heat transfer coefficient are used by an optimization procedure to adjust the thermal field and to match the measured $T_{i}^{\exp }$ and the calculated $T_{i}^{\text {calc }}$ thermal points. This is achieved by minimizing the objective function:

$$
J=\frac{1}{N} \sum_{i=1}^{N}\left(T_{i}^{\text {calc }}-T_{i}^{\exp }\right)^{2}
$$

The used boundary conditions, heat flux and heat transfer coefficient, as well as the calculated thermal field are the results of the inverse method. Thus the inverse method provides the spatial temperature distribution and the local resolution of heat transfer coefficient. The method is therefore able to consider effects like thermal stratification in cooling channels and the local decrease of heat transfer coefficient due to heat transfer deterioration. The comparison of the obtained heat fluxes with a standard calorimetric method shows satisfactory small deviations.

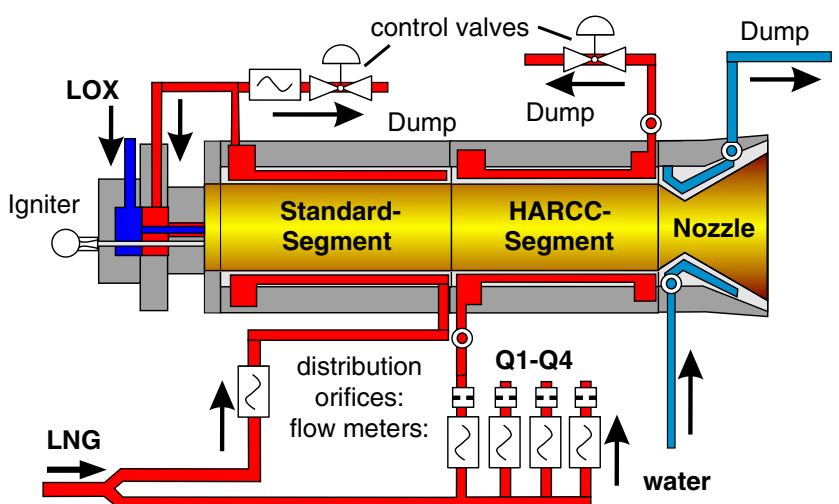

Fig. 4 Distribution of cooling fluids through the combustion chamber.

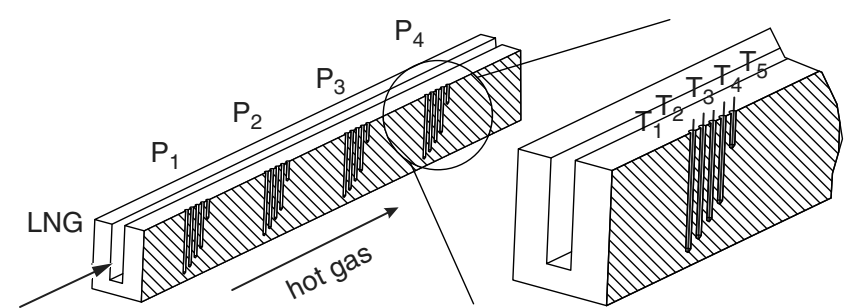

Fig. 5 Positioning of the 20 thermocouples for structural temperature measurements (not to scale).
Table 3 Position of the thermocouples in the structure of the HARCC segment

\begin{tabular}{lccccr}
\hline \hline Axial position & $\mathrm{P} 1$ & $\mathrm{P} 1$ & $\mathrm{P} 3$ & $\mathrm{P} 4$ & \\
\hline Distance from the coolant inlet, $\mathrm{mm}$ & 52 & 85 & 119 & 152 & \\
\hline Radial position & $T_{1}$ & $T_{2}$ & $T_{3}$ & $T_{4}$ & $T_{5}$ \\
\hline Distance from the hot gas side, $\mathrm{mm}$ & 0.7 & 1.1 & 1.5 & 1.9 & 7.5 \\
\hline \hline
\end{tabular}

For the 3D inverse method, a whole cooling channel is modeled, including the inlet and outlet manifolds. The mesh is uniformly spaced with an averaged distance of $0.5 \mathrm{~mm}$ between two nodes, 240.000 nodes in total. For the heat flux at the hot gas side, two parameter are used: one at the beginning and one at the end. Between these values a linear interpolation is used. For the distribution of heat transfer coefficients, two approaches were employed. In the first approach, used for low-aspect-ratio cooling channels Q1 and Q2, a constant heat transfer coefficient along the circumference of the cooling channel is assumed. Thus at the four locations, where the thermocouples are mounted (see also Fig. 5 and Table 3), a heat transfer coefficient is calculated. At the beginning and at the end of the channel, due to a lack of data, constant heat transfer coefficients are assumed. The coolant temperature can be approximated from the heat flux at the hot gas side with the assumption of constant heat capacity at constant pressure $c_{P}$. Effects due to acceleration and compressibility are therefore neglected.

$$
T_{f}(x)=\frac{\int \dot{q}_{w}(x) \mathrm{d} x \cdot L_{\mathrm{HG}}}{c_{P} \cdot \dot{m}}+T_{\mathrm{in}}
$$

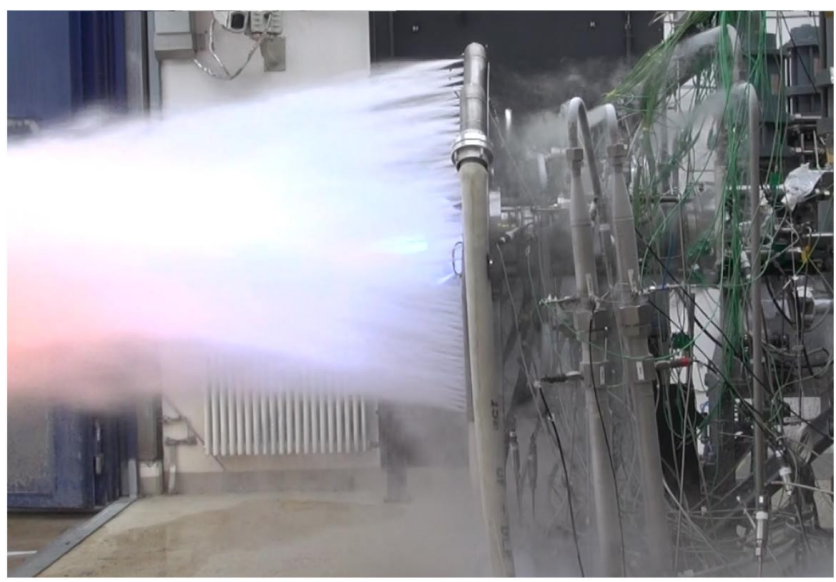

Fig. 6 Picture of the combustion chamber in the test cell and the protecting water shield during a hot run.

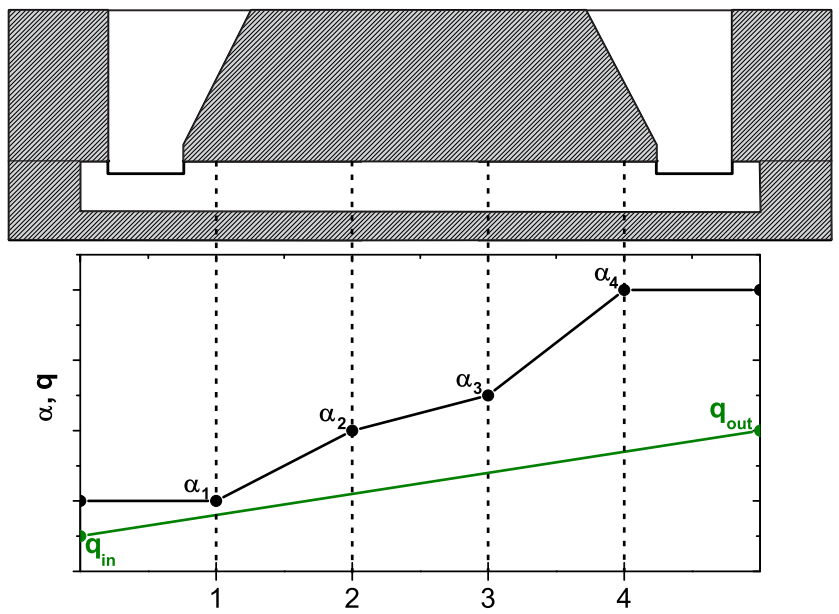

Fig. 7 Boundary conditions for the inverse method (not to scale). 


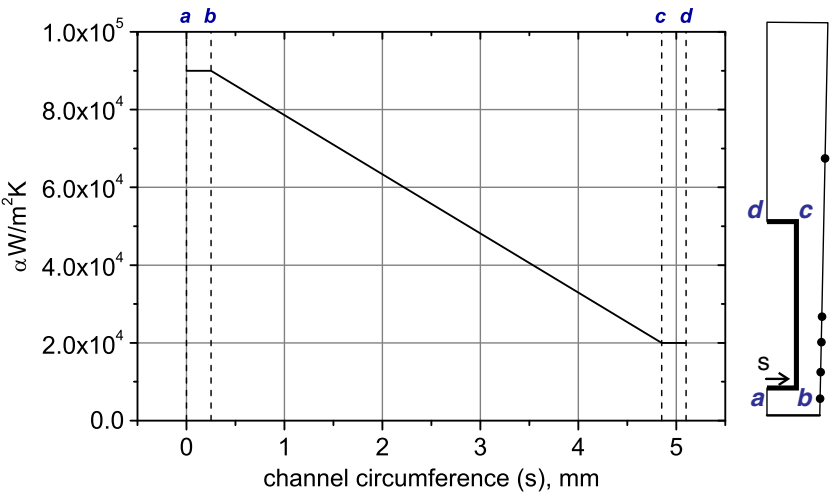

Fig. 8 Sketch of the linear distribution of the heat transfer coefficient for the high-aspect-ratio cooling channel Q4 (not to scale).

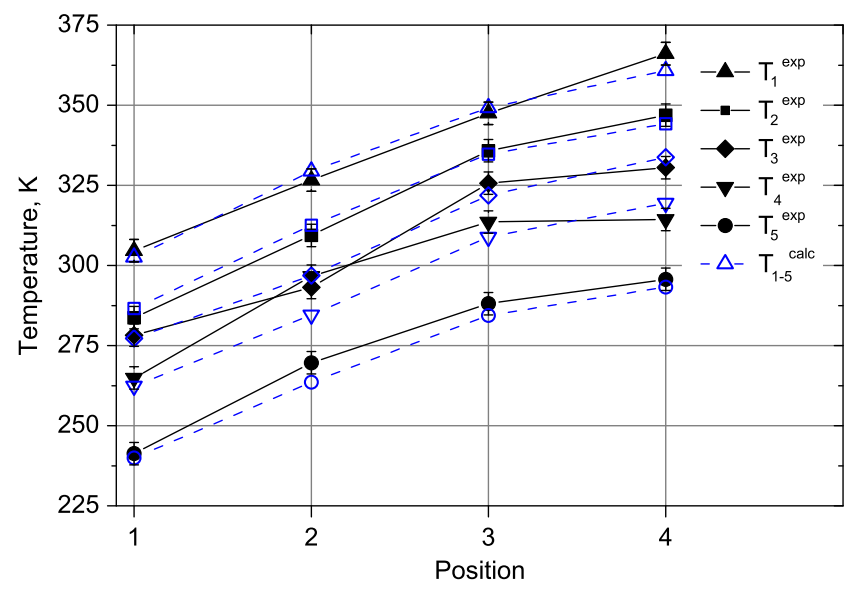

Fig. 9 Example of a match between measured and calculated temperature values (data for quadrant 1 , methane $P_{\mathrm{cc}}=\mathbf{5 0} \mathrm{bar}$, $\dot{m}=20 \mathrm{~g} / \mathrm{s}$ ).

where $\dot{q}_{w}(x)$ refers to the calculated heat flux; $L_{\mathrm{HG}}$ is the hot gas side width per cooling channel, that is, $L_{\mathrm{HG}}=\pi \cdot D_{\mathrm{BK}} / n ; \dot{m}$ is the coolant mass flow; and $T_{\text {in }}$ is the fluid inlet temperature.

For the high-aspect-ratio cooling channels, Q3 and Q4, heat transfer coefficients are set for the bottom and the top of the channel for each of the four axial positions. Linear interpolation is used to obtain changing properties around the circumference of the cooling channel. This approach is sketched in Fig. 8. Thus in these cases eight heat transfer coefficients and two heat fluxes are calculated to determine the thermal field. Additionally, due to the presence of thermal stratification (see Sec. II.C), the fluid temperature at the top of the high-aspect-ratio channels is set to the inlet temperature $T_{\text {in }}$ and a linear distribution of the coolant temperature $T_{f}$ along the channel height is assumed.

Table 4 Achieved measurement accuracy (direct measurements)

\begin{tabular}{lccc}
\hline \hline Device & Pressure sensors & Thermocouple & Measurement turbine \\
\hline Measure & Pressure & Temperature & Volume flow \\
Accuracy & $\pm 0.25 \mathrm{bar}$ & $\pm 2.5 \mathrm{~K}$ & $\pm 1.5 \%$ of measured value \\
\hline \hline
\end{tabular}

An example of the match between measured and calculated temperatures can be seen in Fig. 9. Maximal deviation for this test case is $\left|\left(T_{\text {calc }}-T_{\text {exp }}\right) / T_{\text {exp }}\right| \leq 1.5 \%$. For all other test cases, including the high-aspect-ratio channels, the maximal deviation is $\leq 5 \%$.

Further information about this method can be found in $[17,18]$. In the present study the method is used to calculate the hot gas side wall temperatures. The calculated heat transfer coefficients give insight into the heat transfer especially for high-aspect-ratio cooling channels. According to the definition in Table 1 , the values are used to decide whether a test point is deteriorated or not. However, for the benefit of clarity in the following diagrams, only the temperatures at the four measurement positions are displayed.

\section{Measurement Accuracy}

Table 4 summarizes the errors of the measurement devices, and Table 5 summarizes the error of the derived values. These derived values are calculated based on more than two measurements.

The average heat flux can be calculated by the calorimetric method according to Eq. (3) with the enthalpy difference between inlet $h_{\text {in }}$ and outlet $h_{\text {out }}$ manifold.

$$
\dot{q}_{w}=\frac{\dot{m}}{A}\left(h_{\text {out }}\left(P_{\text {out }}, T_{\text {out }}\right)-h_{\text {in }}\left(P_{\text {in }}, T_{\text {in }}\right)\right)
$$

with

$$
\dot{m}=\rho \cdot \dot{V}
$$

The error of the heat flux calculated with the calorimetric method [Eq. (3)] contains deviations in volume flow $\dot{V}$, density estimation $\rho$, temperature, and pressure at the inlet and outlet manifold. The maximum and minimum values are then calculated with the corresponding maximum and minimum values, with respect to the measurement uncertainties of volume flow, density, pressure, and temperature.

The accuracy of the inverse method depends on the accuracy of the temperature measurement in conjunction with the accuracy of the optimization algorithm. Because data from the 20 thermocouple are used for one calculation, it cannot be said what distribution of single errors leads to the highest or the lowest result. Therefore a random approach was used to estimate the uncertainties of this method. Every measurement data point is randomly loaded with a measurement error according to Table 4 and the inverse method is performed afterward. This procedure is repeated several times, and the difference between maximum and minimum value is seen as the uncertainty of the inverse method.

\section{Results}

Figure 10 shows an overview of the operational field for the current test campaign. The stated mass flow is the sum of the coolant mass flow for the four sectors of the HARCC segment. The pressure inside the cooling channel as well as the heat flux is dependent on the combustion chamber pressure. Therefore these values cannot be evaluated independently from each other. To decouple the heat transfer in the cooling channels from the hot gas conditions inside the combustion chamber, the data are analyzed based on the ratio of heat flux to mass flux $\dot{q}_{w} / G$. In that way, test cases with different combustion chamber pressure and mixture ratio can be compared. Figure 11 displays the operational field in that way for Q1, AR:1.7.

The blue dots in Fig. 11, test cases b1-b4 in Table 6, indicate data points with a constant ratio of $\dot{q}_{w} / G$ and varying coolant pressure values. The average heat flux $\dot{q}_{w}$ is calculated by a standard

Table 5 Achieved measurement accuracy (derived values)

\begin{tabular}{lccccc}
\hline \hline Measure & Mass flow & $\begin{array}{c}\text { Heat flux } \\
\text { (calorimetric) }\end{array}$ & Heat flux (inverse) & $\begin{array}{c}\text { Heat transfer coefficient } \\
\text { (inverse) }\end{array}$ & $\begin{array}{c}\text { Hot gas surface temperature } \\
\text { (inverse) }\end{array}$ \\
\hline $\begin{array}{c}\text { Accuracy } \\
\text { (3.0\% of measured } \\
\text { value }\end{array}$ & $\begin{array}{c} \pm 5 \% \text { of measured } \\
\text { value }\end{array}$ & $\begin{array}{c} \pm 11 \% \text { of measured } \\
\text { value }\end{array}$ & $\pm 19 \%$ of measured value & $\pm 1.5 \%$ of measured value \\
\hline \hline
\end{tabular}




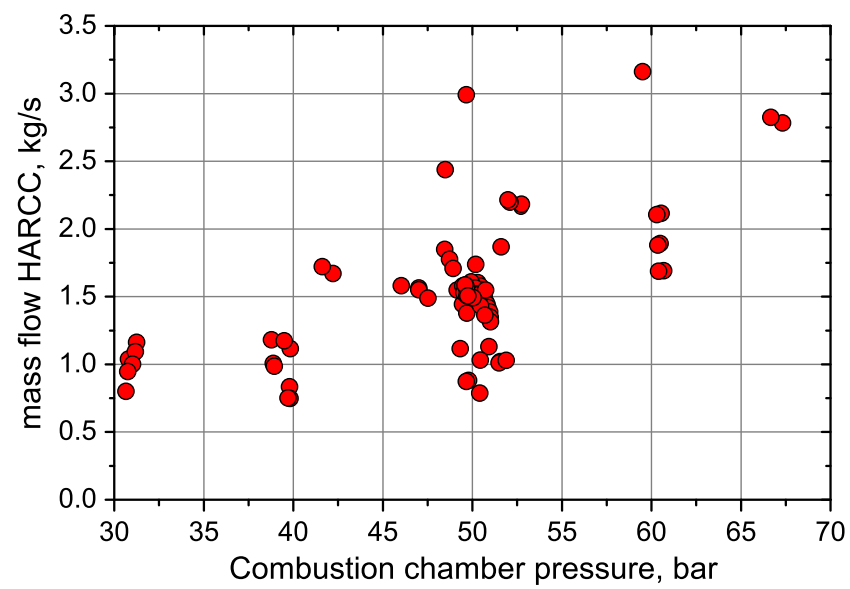

Fig. 10 Test matrix of the current test campaign.

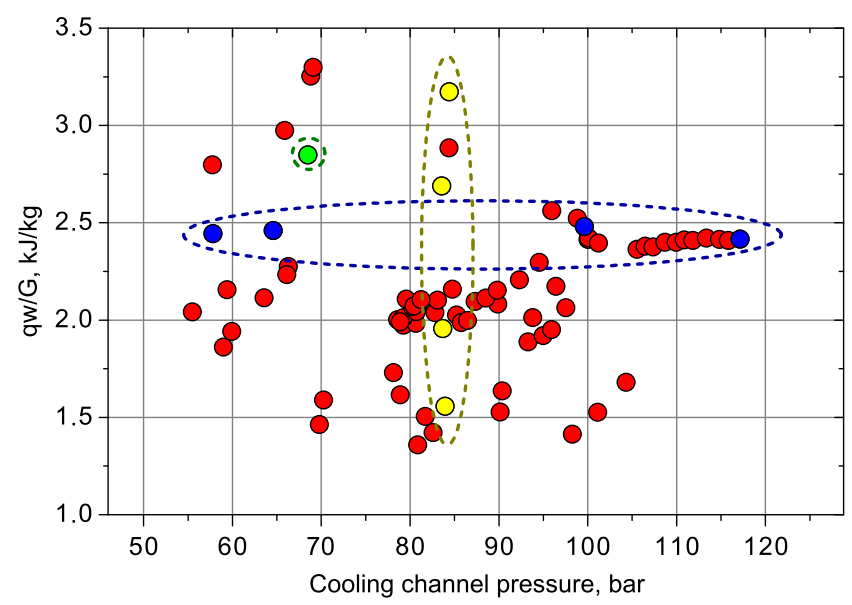

Fig. 11 Test matrix with $\dot{\boldsymbol{q}}_{w} / G$ over $\boldsymbol{P}_{\text {out }}$ and the evaluated test points.

calorimetric method. These points are evaluated in Sec. III.A. The yellow dots, test cases $\mathrm{y} 1-\mathrm{y} 4$ in Table $\underline{6}$, indicate data points with a constant coolant pressure and varying ratio of $\dot{q}_{w} / G$. These points are evaluated in Sec. III.B. Sections III.A and III.B focus on the lowaspect-ratio cooling channel $\mathrm{Q} 1, \overline{A R}=1 . \overline{7 \text {. A }}$ ll different aspect ratios are considered in Sec. III.C. In this section a single test case, green dot in Fig. 11, test case $\mathrm{c} 1$, is evaluated for all geometries.

All data points for all different cooling channels, red dots in Fig. 11, are considered in Sec. III.D. In this section an equation is given to estimate the occurrence of HTD based on the corresponding boundary conditions.

\section{A. Influence of Pressure}

As stated before, the pressure inside the cooling channels, as well as the heat flux, depends on the combustion chamber pressure. This has to be considered when analyzing the influence of pressure. Figure 12 presents the hot gas side wall temperature along the cooling channel length for sector $1, \mathrm{AR}: 1.7$, for a constant $\dot{q}_{w} / G \approx 2.4$ and different pressure values.

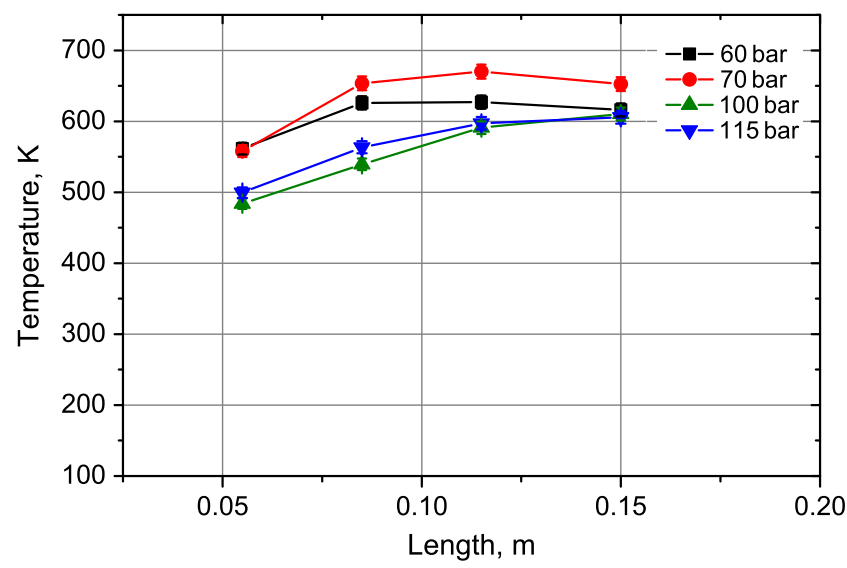

Fig. $12 T_{\mathrm{HG}}$ for $\mathrm{Q1}, \mathrm{AR}: 1.7, \dot{q}_{w} / G \approx 2.4 \mathrm{~kJ} / \mathrm{kg}$, different pressure values.

The figure clearly shows the occurrence of a peak in hot gas surface temperature and therefore heat transfer deterioration for pressures below $P_{\text {in }} \approx 70$ bar, that is $P_{\text {in }} / P_{\text {cr }} \approx 1.5$.

With decreasing pressure, the peak is shifted to the left side, indicating an earlier onset of the deterioration. For the 60-bar case, $\mathrm{b} 1$, the peak in hot gas side wall temperature is at $x=0.1 \mathrm{~m}$ cooling channel length, whereas for the 70-bar case, the peak is at $x=0.125 \mathrm{~m}$. For the higher pressure test cases, $P_{\text {in }} \geq 100 \mathrm{bar}$, no peak is observable, and thus they represent a normal heat transfer. In these cases the wall temperatures are very similar and the heat transfer is independent from the coolant pressure $P_{\text {in }}$.

Pressure, and therefore vicinity to the critical point, is one of the most important factors to describe heat transfer deterioration. The more pronounced the maximum of specific heat at constant pressure gets, the more distinct the separation of gas-like and liquid-like fluid inside the cooling channels.

\section{B. Influence of $\dot{\boldsymbol{q}}_{w} / \boldsymbol{G}$}

After focusing the influence of pressure, and therefore vicinity to the critical point, the effect of heat transfer deterioration is analyzed for different values of $\dot{q}_{w} / G$. Figure 13 shows again the hot gas side wall temperature for Q1, AR:1.7 along the cooling channel length. In Fig. 13, the coolant pressure is kept at a constant value, $P_{\text {out }} \approx 85 \mathrm{bar}$, and the heat flux per mass flux varies between $\dot{q}_{w} / G=1.5 \mathrm{~kJ} / \mathrm{kg}$ and $3.5 \mathrm{~kJ} / \mathrm{kg}$.

For the nondeteriorated cooling fluid, which means all test cases where $\dot{q}_{w} / G \leq 2 \mathrm{~kJ} / \mathrm{kg}$, the figure shows the expected behavior of a rising surface temperature with cooling channel length as well as a rising surface temperature with increasing heat flux per mass flux. When it comes to heat transfer deterioration, test cases $\dot{q}_{w} / G \geq 2.6 \mathrm{~kJ} / \mathrm{kg}$, the wall temperature exhibits a peak that moves forward with increasing heat flux per mass flux $(x=0.125$ for the $\dot{q}_{w} / G=2.6 \mathrm{~kJ} / \mathrm{kg}$ case compared with $x=0.095$ for the $\dot{q}_{w} / G=3.2 \mathrm{~kJ} / \mathrm{kg}$ case).

\section{Influence of the Aspect Ratio}

A single test case was chosen to analyze the influence of different aspect ratios on the heat transfer deterioration. Combustion chamber pressure was $P_{\mathrm{cc}}=39.78$ bar, resulting in heat fluxes of about

Table 6 Boundary conditions for the evaluated test cases

\begin{tabular}{|c|c|c|c|c|c|c|c|c|c|}
\hline Test case & b1 & b2 & b3 & b4 & $\mathrm{y} 1$ & $\mathrm{y} 2$ & y3 & y4 & $\mathrm{c} 1$ \\
\hline$P_{\mathrm{cc}}$, bar & 30.66 & 38.88 & 51.49 & 51.00 & 52.72 & 50.15 & 49.79 & 50.42 & 39.78 \\
\hline$\dot{m}_{\mathrm{HARCC}}, \mathrm{kg} / \mathrm{s}$ & 0.80 & 1.01 & 1.01 & 1.31 & 2.16 & 1.56 & 0.88 & 0.79 & 0.83 \\
\hline$\dot{q}_{w}, \mathrm{MW} / \mathrm{m}^{2}$ & 9.22 & 11.30 & 12.15 & 13.77 & 14.46 & 15.11 & 11.92 & 12.47 & 11.09 \\
\hline$\dot{q}_{w} / G(Q 1), \mathrm{kJ} / \mathrm{kg}$ & 2.44 & 2.46 & 2.48 & 2.42 & 1.56 & 1.96 & 2.69 & 3.17 & 2.85 \\
\hline$P_{\text {in }}$, bar & 60.52 & 69.20 & 102.64 & 120.84 & 92.61 & 90.50 & 85.99 & 88.23 & 71.71 \\
\hline$P_{\text {out }}$, bar & 57.74 & 64.59 & 99.61 & 117.15 & 83.92 & 83.67 & 83.54 & 84.41 & 68.49 \\
\hline$T_{\mathrm{in}}, \mathrm{K}$ & 143.79 & 141.73 & 139.44 & 135.22 & 136.34 & 140.14 & 140.41 & 139.24 & 138.95 \\
\hline
\end{tabular}




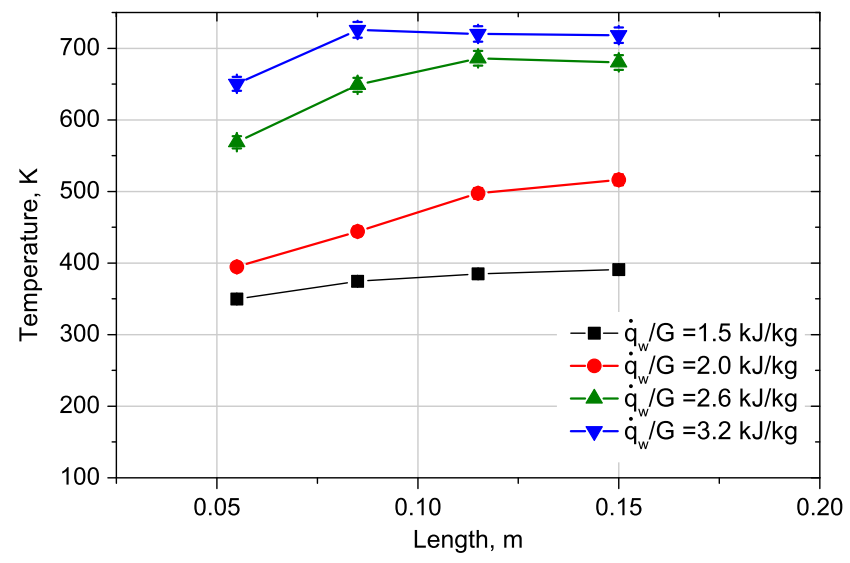

Fig. $13 T_{\mathrm{HG}}$ for Q1, AR:1.7, $P_{\text {out }} \approx 85 \mathrm{bar}$, varying mass flow.

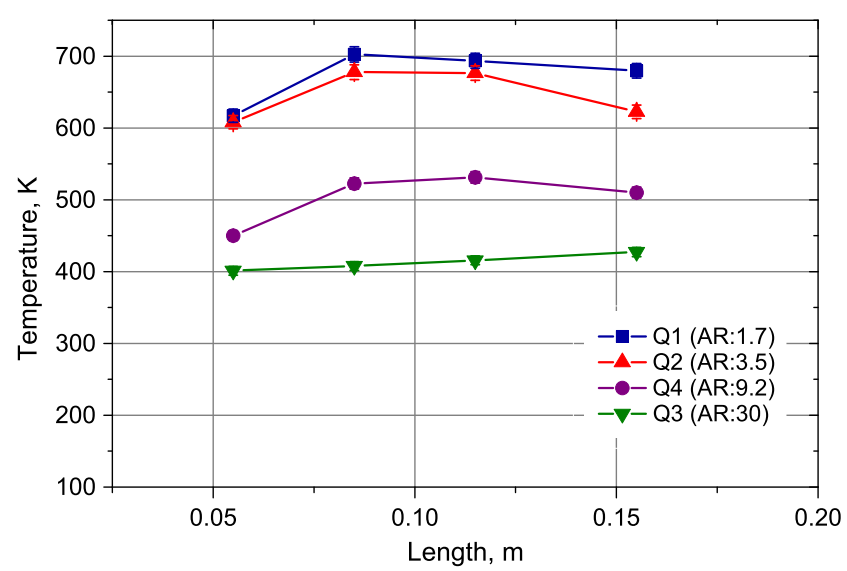

Fig. 14 Hot gas side wall temperatures for different cooling channel geometries.

$\dot{q}_{w} \approx 11 \mathrm{MW} / \mathrm{m}^{2}$. Inlet temperature and pressure was $T_{\text {in }}=139 \mathrm{~K}$ and $P_{\text {in }} \approx 70$ bar, respectively. Coolant mass flow through the entire HARCC segment was $\dot{m}_{\text {HARCC }}=0.8 \mathrm{~kg} / \mathrm{s}$.

Figure 14 shows the hot gas side wall temperature for all four geometries as a function of the cooling channel length. It can easily be seen that the temperature exhibits a distinct maximum for the lowaspect-ratio cooling channels, Q1 \& Q2 (AR:1.7 \& 3.5), and a lesspronounced maximum for the high-aspect-ratio channel, Q4, AR:9.2. The cooling channel with the highest aspect ratio, Q3, AR:30, shows a monotonic increasing temperature, indicating a nondeteriorated trend. Figure 14 also displays a tendency toward a more pronounced maximum at earlier stages in the cooling channel with decreasing aspect ratio.

Similar results were obtained by CFD simulations and can be found, for example, in [19]. This is remarkable because the mass flow per sector is identical and that means less coolant per channel for a higher aspect ratio, because the number of cooling channels increases with increasing aspect ratios, and therefore a higher $\dot{q}_{w} / G$.

The described behavior can also be seen in Fig. 15. This figure presents the temperatures at the measurement point $T_{5}$, that is, $7.5 \mathrm{~mm}$ away from the hot gas side, along the cooling channel length. There is still a distinct maximum visible for the low-aspect-ratio cooling channels Q1 \& Q2, and almost no peak is observable for cooling channel Q4. Measurement point $T_{5}$ is the coldest measurement point and represents a location opposite to the hot gas side point. This result indicates a deterioration for low-aspect-ratio cooling channels at the top and the bottom and accordingly also on the side of the channels.

Thermal stratification: For cooling channels with a very high aspect ratio, the problem of thermal stratification is known. Because of a limited mixing in these channels, hot cooling fluid remains at the

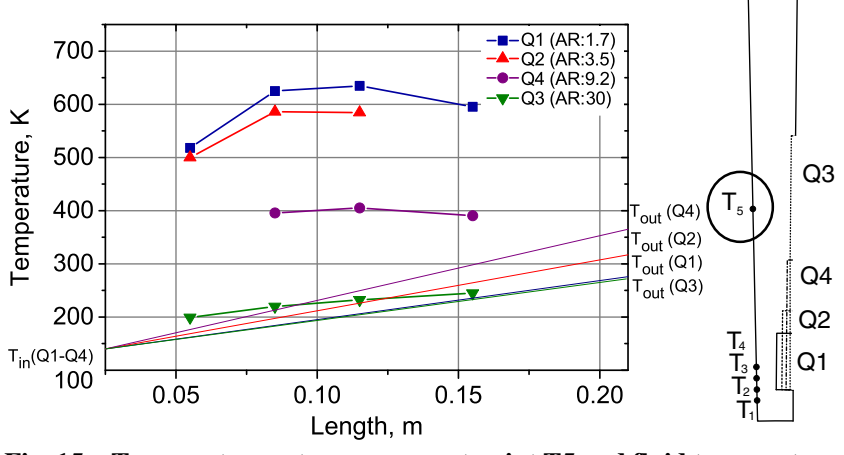

Fig. 15 Temperatures at measurement point $\mathrm{T} 5$ and fluid temperature for different cooling channel geometries.

bottom part and cold fluid remains at the top part of the channels $[14,20,21]$. In Fig. 15 the fluid temperatures at the inlet $T_{\text {in }}(Q 1-Q 4)$ and outlet manifold $T_{\text {out }}(Q 1)-T_{\text {out }}(Q 4)$ are displayed and connected with a straight line. Thus this line roughly represents the mean fluid temperature. For cooling channel Q3, the temperature of measurement point $T_{5}$ is very close to the mean fluid temperature, indicating a strong thermal stratification and therefore a lower fluid temperature at the top part of the channel and a higher temperature at the lower part.

Another quantitative assessment is to consider the ratio of minimum to maximum coolant side wall temperature. This ratio is summarized in Table 7 for the four sectors at measurement position 4 . With increasing aspect ratio this value decreases. For the high-aspect-ratio channels Q3 \& Q4 this ratio is low, and therefore thermal stratification is high.

Because of this fluid temperature distribution, HTD may only occur at the lower part of the cooling channel but a normal nondeteriorated heat transfer is present at the top and upper side part of the channel. This effect is intensified by the fact that the heat flux through the combustion chamber wall decreases in the radial direction. This is advantageous in the top part of the channel because it lowers the ratio of $\dot{q}_{w} / G$ and therefore the occurrence of heat transfer deterioration. This mechanism is noticeable in the heat flux distribution.

Figure 16 shows the heat flux along the cooling channel circumference for cooling channel Q4, AR: 9.2, for all 4 measurement positions. These values are computed directly by the inverse method based on the calculated heat transfer coefficient and the coolant temperature. It can be seen that the progress of heat flux around the circumference changes from a decreasing, Pos. 1, to a rising trend, Pos. 2-4, when it comes to heat transfer deterioration. In that picture Pos. 1 represents nondeteriorated behavior; that is, the amount of heat that is transferred decreases along the radial direction. When it comes to heat transfer deterioration, Pos. $2-4$, the trend is inverted. The amount of heat that is transferred rises along the radial direction. This behavior illustrates that in high-aspect-ratio channels, HTD only lowers heat transfer capabilities in the lower part of the channel but does not influence heat transfer in the upper part. With increasing aspect ratio, the ratio of cooling channel area that is affected by HTD is decreasing and accordingly the effect is vanishing.

\section{Boundary Between HTD and Non-HTD}

All data points for the four sectors are summarized in Fig. 17. The data points are plotted as a function of reduced pressure, $P_{\text {in }} / \overline{P_{\mathrm{cr}}}$, and heat flux per mass flow per area, $\dot{q}_{w} / G$. They reveal whether HTD was detected or not. Based on the definition in Table 1, a test case is considered deteriorated when a peak in hot gas side wall temperature occurs at the same location where the heat transfer coefficient

Table 7 Ratio of minimal to maximal coolant side wall temperature at measurement position 4

\begin{tabular}{lcccc}
\hline \hline Sector & Q1 (AR:1.7) & Q2 (AR:3.5) & Q4 (AR:9.2) & Q3 (AR:30) \\
\hline$T_{\min } / T_{\max }$ & 0.90 & 0.88 & 0.68 & 0.61 \\
\hline \hline
\end{tabular}




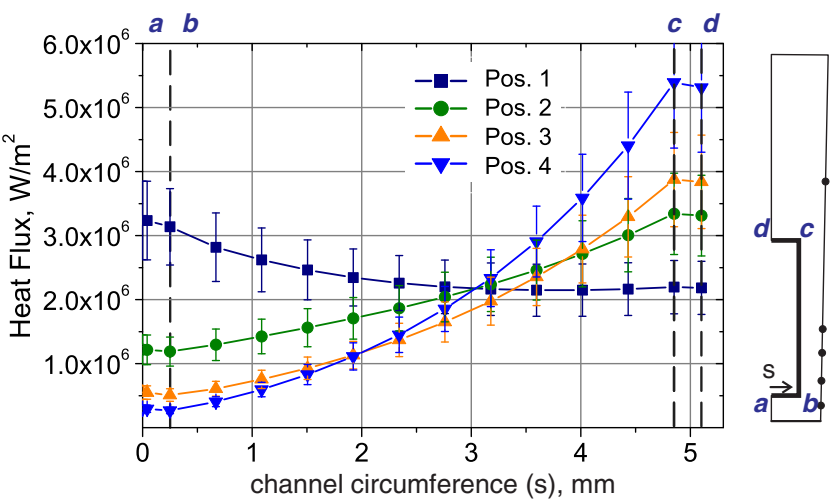

Fig. 16 Heat flux into the cooling channel as function of cooling channel circumference for the 4 measurement positions. Q4, AR: 9.2.

exhibits a minimum value. It can clearly be seen that heat transfer deterioration occurs in cooling channels when the pressure is close to the critical point and when $\dot{q}_{w} / G$ is high.

The ratio between the heat flux and the mass flow per area, $\dot{q}_{w} / G$, is considered in the literature as an indicator whether HTD occurs or not. It is expected that if this ratio is higher than a threshold value, $\left(\dot{q}_{w} / G\right)>\left(\dot{q}_{w} / G\right)_{\mathrm{tr}}$, the heat transfer will be deteriorated. In [8] $\left(\dot{q}_{w} / G\right)_{\text {tr }}$ is considered to be a linear function of the inlet pressure:

$$
\left(\frac{\dot{q}_{w}}{G}\right)_{\mathrm{tr}}=43.2 \cdot 10^{-6} \cdot P_{\mathrm{in}}+31.4
$$

with $\left(\dot{q}_{w} / G\right)_{\text {tr }}$ in $[\mathrm{J} / \mathrm{kg}]$ and $P_{\text {in }}$ in $P a$.

Equation (5) was obtained for a smooth circular tube that is uniformly heated. In the present study rectangular channels are
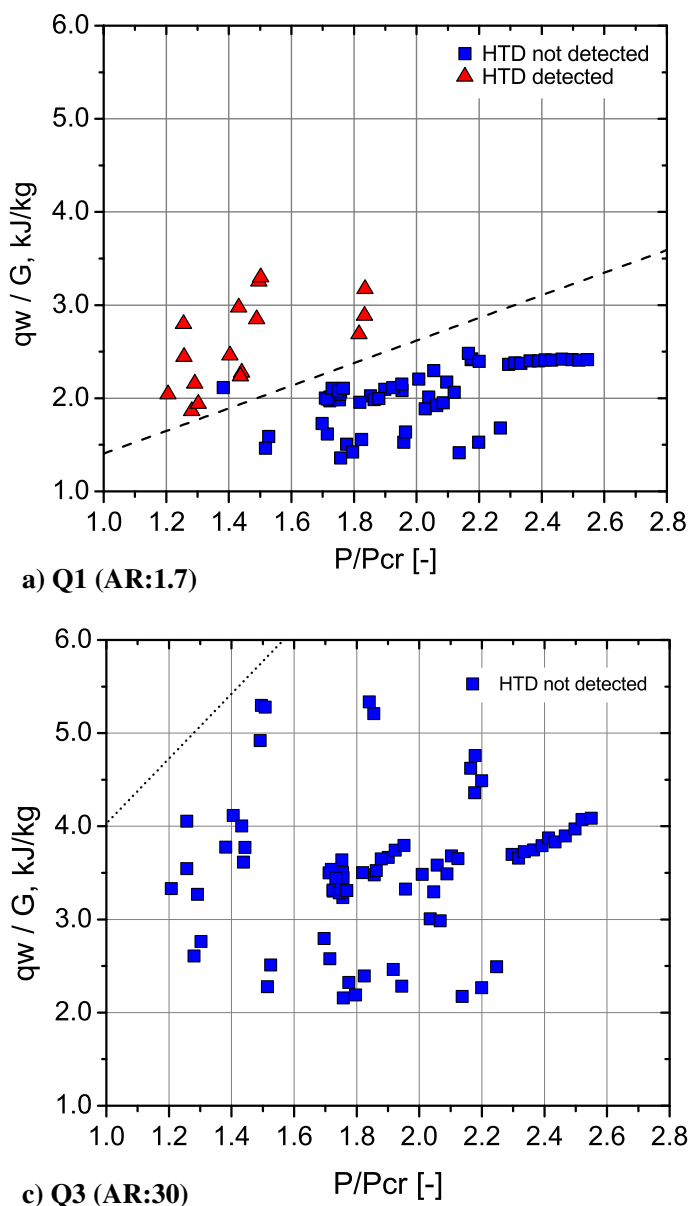

heated from one side only. Therefore the equation was slightly modified by a factor of 2.4 and expanded by the ratio of coolant side area to heated area $U / L_{\mathrm{HG}}$. $U$ is the cooling channel circumference, that is, $U=2 \cdot(h+b) . L_{\mathrm{HG}}$ is the hot gas side width per cooling channel, calculated as $L_{\mathrm{HG}}=\pi \cdot D_{\mathrm{BK}} / n$.

$$
\left(\frac{\dot{q}_{w}}{G}\right)_{\mathrm{tr}}=\left(43.2 \cdot 10^{-6} \cdot P_{\mathrm{in}}+31.4\right) \frac{U}{L_{\mathrm{HG}}} \cdot 2.4
$$

Equation (6) is plotted as a dashed line in Fig. 17. For the highaspect-ratio cooling channel $\mathrm{Q} 3, A R=30$, the equation is out of scope, encouraging the assumption that this high-aspect-ratio cooling channel is less prone to heat transfer deterioration. For the highaspect-ratio cooling channel Q4, $A R=9.2$, Eq. (6) is slightly too high. This deviation can be explained with the aforementioned thermal stratification. The whole cooling channel area is not available for heat transfer. If we assume an effective available area of $80 \%$ we get the dotted line in Fig. 17. For Q3 an effective available area of $65 \%$ was assumed to get the equivalent dotted line. With that exception for high-aspect-ratio cooling channels, Eq. (6) predicts $>98 \%$ of all test points correct. The test points that exhibit HTD are above the threshold value and the other points are below.

\section{Conclusions}

A systematic experimental analysis of transcritical methane in cooling channels of rocket combustion chambers at real conditions was performed. Clear experimental evidence of heat transfer deterioration for conditions close to the critical point was found. Particularly in cooling channels with a low aspect ratio, a distinct maximum in hot gas wall temperature arises, combined with a loss in heat transfer.

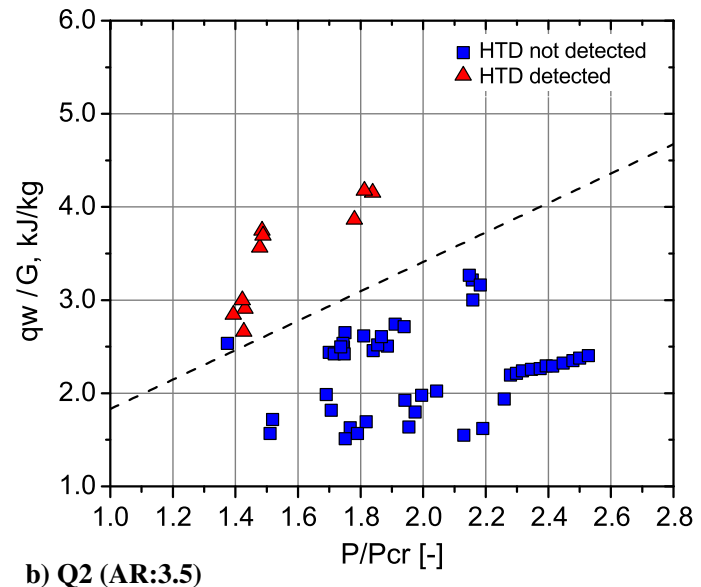

b) $Q 2(A R \cdot 3.5)$

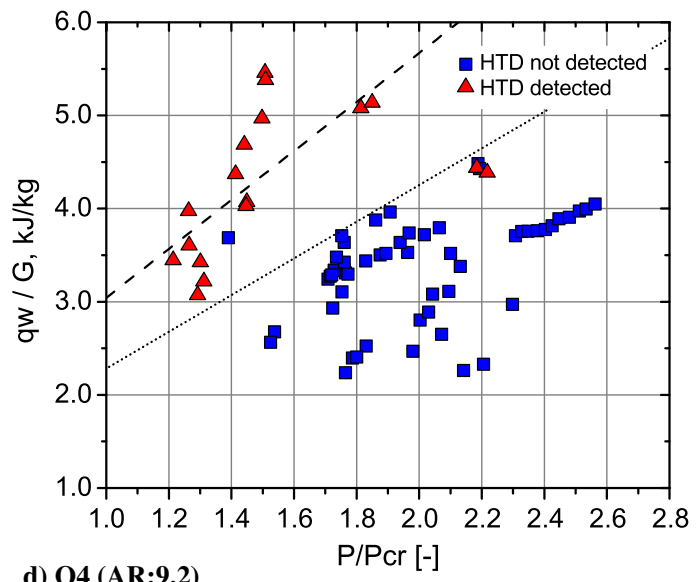

d) Q4 (AR:9.2)

Fig. 17 Data points for all aspect ratios reveal whether HTD was detected or not. 
The evaluation of the obtained data indicates that heat transfer deterioration primarily occurs in cooling channels with a low aspect ratio. The higher area for the heat transfer as well as the well-known and pronounced thermal stratification within high-aspect-ratio cooling channels appears to be advantageous and, at least in this study, heat transfer deterioration seems to be a problem primarily for low-aspect-ratio cooling channels.

In addition, the data reveal the influence of pressure as well as heat flux per mass flux on the problem of heat transfer deterioration. A simple formula that describes the boundary of a nondeteriorated fluid to a deteriorated one based on these parameter is given.

Further parameters that are known to have an influence on heat transfer and particularly on the problem of heat transfer deterioration could not be analyzed with the present setup. These are, for example, roughness inside the cooling channels, combustion chamber wall material, heat flux profiles, or curved cooling channels.

\section{Acknowledgments}

The authors would like to thank the P8 test bench crew for their help and support during the successful test campaign.

\section{References}

[1] Rudnykh, M., Carapellese, S., Liuzzi, D., Arione, L., Caggiano, G., Bellomi, P., D'Aversa, E., Pellegrini, R., Lobov, S., and Gurtovoy, A., et al., "Development of LM10-MIRA LOX/LNG Expander Cycle Demonstrator Engine," Acta Astronautica, Vol. 126, Sept.-Oct. 2016, pp. 364-374. doi:10.1016/j.actaastro.2016.04.018

[2] Börner, M., Manfletti, C., Hardi, J., Suslov, D., Kroupa, G., and Oschwald, M., "Laser Ignition of a Multi-Injector LOX/Methane Combustor," CEAS Space Journal, Vol. 10, No. 2, 2018, pp. 273-286. doi:10.1007/s12567-018-0196-6

[3] Banuti, D. T., "Crossing the Widom-Line-Supercritical PseudoBoiling," The Journal of Supercritical Fluids, Vol. 98, No. 03, 2015, pp. 12-16. doi:10.1016/j.supflu.2014.12.019

[4] Kurganov, V., Zeigarnik, Y., and Maslakova, I., "Heat Transfer and Hydraulic Resistance of Supercritical-Pressure Coolants. Part I: Specifics of the Thermophysical Properties of Supercritical Pressure Fluids and Turbulent Heat Transfer Under Heating Conditions in Round Tubes (State of the Art)," International Journal of Heat and Mass Transfer, Vol. 55, Nos. 11-12, 2012, pp. 3061-3075. doi:10.1016/j.ijheatmasstransfer.2012.01.031

[5] Urbano, A., and Nasuti, F., "Parametric Analysis of Cooling Properties of Candidate Expander-Cycle Fuels," Journal of Propulsion and Power, Vol. 30, No. 1, Jan. -Feb. 2014, pp. 153-163. doi:10.2514/1.B34852

[6] Pizzarelli, M., "A CFD-Derived Correlation for Methane Heat Transfer Deterioration," Numerical Heat Transfer, Part A 2016, Vol. 69, No. 3, 2015, pp. 242-264. doi:10.1080/10407782.2015.1080575

[7] Shiralkar, B. S., and Griffith, P., "Deterioration in Heat Transfer to Fluids at Supercritical Pressure and High Heat Fluxes," Journal of Heat Transfer, Vol. 91, No. 1, 1969, pp. 27-36. doi:10.1115/1.3580115

[8] Urbano, A., and Nasuti, F., "Onset of Heat Transfer Deterioration in Supercritical Methane Flow Channels," Journal of Thermophysics and
Heat Transfer, Vol. 27, No. 2, April-June 2013, pp. 298-308. doi: 10.2514/1.T4001

[9] Gu, H., Li, H., and Luo, Y., "Experimental Investigation on Convective Heat Transfer from a Horizontal Miniature Tube to Methane at Supercritical Pressures," Applied Thermal Engineering, Vol. 58, Nos. 1-2, Sept. 2013, pp. 490-498. doi:10.1016/j.applthermaleng.2013.04.049

[10] Votta, R., Battista, F., Salvatore, V., Pizzarelli, M., Leccese, G., Nasuti, F., and Meyer, S., "Experimental Investigation of Transcritical Methane Flow in Rocket Engine Cooling Channel," Applied Thermal Engineering, Vol. 101, May 2016, pp. 61-70. doi:10.1016/j.applthermaleng.2015.12.019

[11] Pizzarelli, M., "The Status of the Research on the Heat Transfer Deterioration in Supercritical Fluids: A Review," International Communications in Heat and Mass Transfer, Vol. 95, July 2018, pp. 132-138. doi:10.1016/j.icheatmasstransfer.2018.04.006

[12] Linstrom, P., and Mallard, W. G., "NIST Chemistry WebBook, NIST Standard Reference Database Number 69," National Inst. of Standards and Technology, Gaithersburg, MD, http://webbook.nist.gov [retrieved 07 Nov. 2015].

[13] Urbano, A., and Nasuti, F., "Parametric Analysis of Heat Transfer to Supercritical-Pressure Methane," Journal of Thermophysics and Heat Transfer, Vol. 26, No. 3, July-Sept. 2012, pp. 450-463. doi:10.2514/1.T3840

[14] Woschnak, A., "Untersuchung des Wärmeübergangs in regenerativ gekühlten Schubkammern kryogener Raketentriebwerke," Ph.D. Thesis, RWTH Aachen Univ., Shaker Verlag GmbH, Aachen, 2009.

[15] Kuhl, D., Holzer, A., and Haidn, O. J., "Computational Solution of the Inverse Heat Conduction Problem of Rocket Combustion Chambers," 35th AIAA/ASME/SAE/ASEE Joint Propulsion Conference \& Exhibit, AIAA Paper 1999-2913, 1999. doi:10.2514/6.1999-2913

[16] Suslov, D., Woschnak, A., Greuel, D., and Oschwald, M., "Measurement Techniques for Investigation of Heat Transfer Processes at European Research and Technology Test Facility P8," 1st EUCASS, 2005-07-042005-07-07, 2005.

[17] Haemisch, J., Suslov, D., and Oschwald, M., "Experimental Analysis of Heat Transfer Processes in Cooling Channels of a Subscale Combustion Chamber at Real Thermal Conditions for Cryogenic Hydrogen and Methane," 6th Space Propulsion Conference, Sevilla, May 2018.

[18] Oschwald, M., Suslov, D., Haemisch, J., Haidn, O., Celano, M. Kirchberger, C., Rackemann, N., Preuss, A., and Wiedmann, D., "Measurement of Heat Transfer in Liquid Rocket Combustors," High Pressure Flows for Propulsion Applications, AIAA Progress in Astronautics and Aeronautics Series (accepted for publication).

[19] Arun, M., Akhil, J., Noufal, K., Baby, R., Babu, D., and Prakash, M. J., "Effect of Aspect Ratio on Supercritical Heat Transfer of Cryogenic Methane in Rocket Engine Cooling Channels," Frontiers in Heat and Mass Transfer, Vols. 8-23, 2017. doi:10.5098/hmt.8.23

[20] Kacynski, K. J., "Thermal Stratification Potential in Rocket Engine Coolant Channels," NASATR TM-4378, E-6135, NAS 1.15:4378, Lewis Research Center, Cleveland, OH, 1992.

[21] Pizzarelli, M., Nasuti, F., and Onofri, M., "Trade-Off Analysis of HighAspect-Ratio-Cooling-Channels for Rocket Engines," International Journal of Heat and Fluid Flow, Vol. 44, Dec. 2013, pp. 458-467. doi:10.1016/j.ijheatfluidflow.2013.08.003

J. M. Seitzman Associate Editor 